\title{
Evaluation of economic recovery capability under the background of COVID-19: application of a big data information processing model
}

\author{
Yufeng Shi ${ }^{*}$, Jiaohui Tang, and Dan Wan \\ ${ }^{1}$ School of Yueshang/MBA, Guangdong University of Finance \& Economics, Guangzhou, China
}

\begin{abstract}
In order to promote local economic recovery under the COVID19 pandemic, this paper first constructs a big data information processing model, then measure the growth of GDP and fiscal revenue driven by consumption, investment and export in Guangdong province. The measurement results show that under the stimulus of consumption, investment and export of the same intensity, Guangzhou, Shenzhen and Foshan perform better in the GDP index, while Shenzhen, Shaoguan and Qingyuan perform better in the fiscal revenue index. To this end, local governments should give priority to Guangzhou, Shenzhen, Foshan, Shaoguan and Qingyuan to take effective economic stimulus measures to achieve faster growth in GDP and fiscal revenue. The research results are of practical significance for guiding local governments to implement precise policies to promote the process of economic recovery.
\end{abstract}

Keywords: COVID-19, Local economy, Measurement model.

\section{Introduction}

At the beginning of 2020, affected by the COVID-19, China's economy was in a stagnant or semi-stagnant state in many fields, leading to a year-on-year drop of $6.8 \%$ in GDP and $14.3 \%$ in fiscal revenue in the first quarter. At present, China has made initial achievements in the control of the COVID-19 and the economy has begun to recover, while the preliminary statistics show that the GDP growth rate in 2020 is only $2.3 \%$, much lower than the average annual growth rate of $9.0 \%$ since the reform and opening up, making it the lowest GDP growth rate since 1978. With the spread of the epidemic in the world, China's foreign trade, foreign investment and other economic activities have been greatly impacted, which has brought greater challenges to the sustainable and healthy development of China's economy.

Chinese scholars have responded positively to the urgent need of economic recovery under the current situation of COVID-19, and carried out theoretical and practical discussions on the core topics of COVID-19's influence on the economy and the corresponding measures to promote economic recovery. Gengzhong Feng et al (2020) expounds the impact of epidemic situation on the integrity of supply chain from the perspective of its essential attribute.Qiaobin Feng et al (2020) assessed the negative impact of the epidemic on fiscal

* Corresponding author: shiyufeng@gdufe.edu.cn 
revenue. Ziqiang Xin et al (2020)studied the impact of the epidemic on public confidence, value judgment, willingness to spend. Yangjie Wang et al (2020) explained the development opportunities and countermeasures of China's digital economy industry under the Covid-19 epidemic situation from the perspective of industrial upgrading. Beijing (2020) creatively extracted the concept of organizational capital from the practice of the national system in dealing with the epidemic situation, and elaborated that China's economy has incomparable institutional advantages and economic characteristics in terms of organizational capital. The existing literature mainly discusses from the macro level, but does not discuss the heterogeneity of local economic recovery and the accuracy of policy measures from the micro perspective.

Starting from the micro operation level and focusing on the capability of local economic recovery, this paper carries out research on the measurement model and empirical test. The specific chapter structure is as follows:

The second chapter is to construct the measurement model. Based on the DEA models, this paper constructs the Recovery Capability Index model (RCI) to overcome the problems such as the inaccuracy and the cumbersome solution of the DEA models (Haixia Lan et al, 2020 and Zhanxin Ma et al, 2019).

The third chapter is empirical study. This paper selects 21 prefecture-level cities in Guangdong province as the research objects, and studies the five panel data of consumption, investment, export, GDP and fiscal revenue of each city from 2012 to 2020.

The main innovations of this paper are as follows: I. The RCI model constructed in this paper has the characteristics of clear economic significance, simple calculation and accurate measurement. It can be used as a powerful tool to deal with big data information. II. The conclusions of this paper can provide a theoretical basis for local governments and help them to accurately implement policies to revive the economy.

\section{Information processing model}

\subsection{Classical DEA}

Classical DEA was proposed by Chames et al (1978), and after continuous improvement, many derivative forms of DEA have been proposed one after another, forming a DEA family ( Banker R D et al, 1984; Charnes A et al, 1987; Sengupta J K, 1987; Seiford L M et al, 1990; Sengupta J K, 1999; Lovell C A K, 2003; Cullinane K et al, 2004), which are widely used in efficiency evaluation. The classical DEA mathematical expression is as follows:

$$
\text { Objective function: } \min E_{s}
$$

Input constraints:

$$
\sum_{k=1}^{K} u_{\alpha, k} \mathrm{w}_{k}^{s} \leq u_{\alpha, \mathrm{s}} \mathrm{E}_{\mathrm{s}}
$$

Output constraints:

$$
\sum_{k=1}^{K} v_{\beta, k} \mathrm{~W}_{k}^{s} \geq v_{\beta, \mathrm{s}}
$$

Among them, $\alpha, \beta, k, s, K$ respectively represent input variable, output variable, production unit, evaluated production unit and the number of units. $w_{k}^{s}$ represents the 
weighting coefficient of the input (output) variable of unit $k$ when evaluating the unit $S$ $\left(\sum_{k=1}^{K} \mathrm{w}_{k}^{s}=1\right) . E_{s}$ represents the efficiency factor of the unit $s \quad\left(0 \leqslant E_{s} \leqslant 1\right.$, the larger the value, the higher the efficiency). $u_{\alpha, k}$ represents the value of the unit $k$ when the input variable is $\alpha . v_{\beta, k}$ represents the value of the $k$ when the output variable is $\beta$.

\subsection{The construction of $\mathrm{RCI}$}

\subsubsection{The first step is dynamic multidimensional optimization}

The objective function of the classic DEA is dynamically expanded by multiple factors, the constraint are redefined, so that the original static single-factor model is converted into a dynamic multi-factor model.

Objective function:

$$
\min \sum_{t=1}^{T}\left(\sum_{\alpha=1}^{m} E_{\alpha, s, t}+\sum_{\beta=1}^{n} E_{\beta, s, t}\right)
$$

Input constraints:

$$
E_{\alpha, \mathrm{s}, t}=\sum_{k=1}^{K-1} u_{\alpha, k, t} \mathrm{w}_{k, t}^{s} / u_{\alpha, \mathrm{s}, t}
$$

Output constraints:

$$
E_{\beta, \mathrm{s}, t}=v_{\beta, \mathrm{s}, t} / \sum_{k=1}^{K-1} v_{\beta, k, t} \mathrm{~W}_{k, t}^{s}
$$

Among them, $\alpha, \beta, k, s, K$ are same as above, $t$ represents the measurement period, $w_{k, t}^{s}$ represents the weighting coefficient in the period of $t\left(\sum_{k=1}^{K-1} \mathrm{~W}_{k, t}^{s}=1\right)$. $u_{\alpha, s, t}$ represents the value of the unit $s$ when the input variable is $\alpha$ in the period of $t$. $u_{\alpha, k, t}$ represents the value of the unit $k$ when the input variable is $\alpha$ in the period of $t$. $E_{\alpha, s, t}$ represents the efficiency factor of the unit $s$ when the input variable is $\alpha$ in the period of $t . v_{\beta, s, t}$ represents the value of the $s$ when the output variable is $\beta$ in the period of $t . v_{\beta, k, t}$ represents the value of the unit $k$ when the output variable is $\beta$ in the period of $t . E_{\beta, s, t}$ represents the efficiency factor of the unit $s$ when the output variable is $\beta$ in the period of $t$.

\subsubsection{The second step is to define the recovery index}

Based on the above formulas (4), (5) and (6), let: 


$$
I_{\beta, s, t}=E_{\beta, s, t} \sum_{\alpha=1}^{m} E_{\alpha, s, t}
$$

Formula (7) can be used to measure the recovery capability of local GDP and fiscal revenue driven by consumption, investment and export. The values 1, 2 and 3 of $\alpha$ respectively represent the economic recovery measures of consumption, investment, export, and the values 1 and 2 of $\beta$ respectively represent the economic indicators of GDP and fiscal revenue. Then, according to formulas (5) and (6), $I_{\beta, s, t}$ can be transformed into the following expression:

$$
I_{\beta, s, t}=\sum_{\alpha=1}^{3} \frac{\mathrm{v}_{\beta, s, t} / u_{\alpha, s, t}}{\sum_{k=1}^{K-1} w_{k, t}^{s} v_{\beta, k, t} / \sum_{k=1}^{K-1} w_{k, t}^{s} u_{\alpha, k, t}}
$$

Among them, $\quad \mathrm{v}_{\beta, s, t} / u_{\alpha, s, t}$ and $\quad \sum_{k=1}^{K-1} w_{k, t}^{s} v_{\beta, k, t} / \sum_{k=1}^{K-1} w_{k, t}^{s} u_{\alpha, k, t}$ respectively represent the conversion ratio and the weighted conversion ratio between any of the three economic recovery measures and the two economic indicators. $\left(\mathrm{v}_{\beta, s, t} / u_{\alpha, s, t}\right) /\left(\sum_{k=1}^{K-1} w_{k, t}^{s} v_{\beta, k, t} / \sum_{k=1}^{K-1} w_{k, t}^{s} u_{\alpha, k, t}\right)$ and $\sum_{\alpha=1}^{3} \frac{\mathrm{v}_{\beta, s, t} / u_{\alpha, s, t}}{\sum_{k=1}^{K-1} w_{k, t}^{s} v_{\beta, k, t} / \sum_{k=1}^{K-1} w_{k, t}^{s} u_{\alpha, k, t}}$ respectively represent the substitution coefficient of the conversion ratio and its sum between any of the three economic recovery measures and the two economic indicators of $s$ city in the year of $t$. The economic significance of $I_{\beta, s, t}$ is the recovery indexes of local GDP and fiscal revenue driven by consumption, investment, export of $s$ city in the year of $t$, which is used to measure the recovery capability of GDP and fiscal revenue. The greater the value of the index, the greater the recovery capability; conversely, the less capability for recovery.

\subsubsection{The third step is the application of $\mathrm{RCl}$}

When $\beta$ is equal to 1 , if $\left(\mathrm{v}_{1, s, t} / u_{1, s, t}\right) /\left(\sum_{k=1}^{K-1} w_{k, t}^{s} v_{1, k, t} / \sum_{k=1}^{K-1} w_{k, t}^{s} u_{1, k, t}\right) \geq 1$, it means that the conversion ratio between any of the three economic recovery measures and GDP of $s$ city is the highest, and any combination scheme of other cities cannot replace city $s$ (The weight of $w_{k, t}^{s}$ is the most unfavorable solution to s city), then:

$$
I_{1, s, t}=\sum_{\alpha=1}^{3} \frac{\mathrm{v}_{1, s, t} / u_{\alpha, s, t}}{\sum_{k=1}^{K-1} w_{k, t}^{s} v_{1, k, t} / \sum_{k=1}^{K-1} w_{k, t}^{s} u_{\alpha, k, t}} \geq 3
$$

It means that the conversion ratio between the three economic recovery measures and GDP of $s$ city is the highest. Therefore, this paper takes 3 as the critical value of $I_{1, s, t}$. If the GDP recovery index of a region is greater than 3 , it means that the GDP of the region has high recovery capability, on the contrary, it does not have high recovery capability. 
Through the above three steps, the recovery capability index model (RCI) is formed. The differences between this model and DEA model are shown in the following table:

Table 1. Comparison between RCI and DEA

\begin{tabular}{|c|c|c|}
\hline Model & RCI & DEA \\
\hline Structure & $\begin{array}{c}\text { Dynamic multidimensional index } \\
\text { Equality constraints }\end{array}$ & $\begin{array}{c}\text { Single index } \\
\text { Inequality constraints }\end{array}$ \\
\hline Application & $\begin{array}{c}\text { High precision } \\
\text { Easy to solve }\end{array}$ & $\begin{array}{c}\text { Low precision } \\
\text { Difficult to solve }\end{array}$ \\
\hline
\end{tabular}

\section{Empirical study}

\subsection{Operating procedure}

This paper selects five panel data, namely total retail sales of consumer goods, investment in fixed assets, foreign export trade, GDP and general public financial budget revenue of 21 prefecture-level cities in Guangdong Province from 2012 to 2020, as the alternative indicators in consumption, investment, export, GDP and fiscal revenue of each city.( The data is from the statistical yearbook of Guangdong Province from 2012 to 2020 )

The first step is to solve equations (4), (5) and (6) with the help of nonlinear programming solution software; The second step is to calculate the GDP recovery index and fiscal revenue recovery index of 21 cities from 2012 to 2019 according to Equation (7) based on the optimal solution (the calculation results are shown in Figure 1 and Figure 2); The third step is to select cities with high recovery capability according to formula (9) for retrospective analysis.

\subsection{Empirical results}

\subsubsection{The GDP recovery capability}

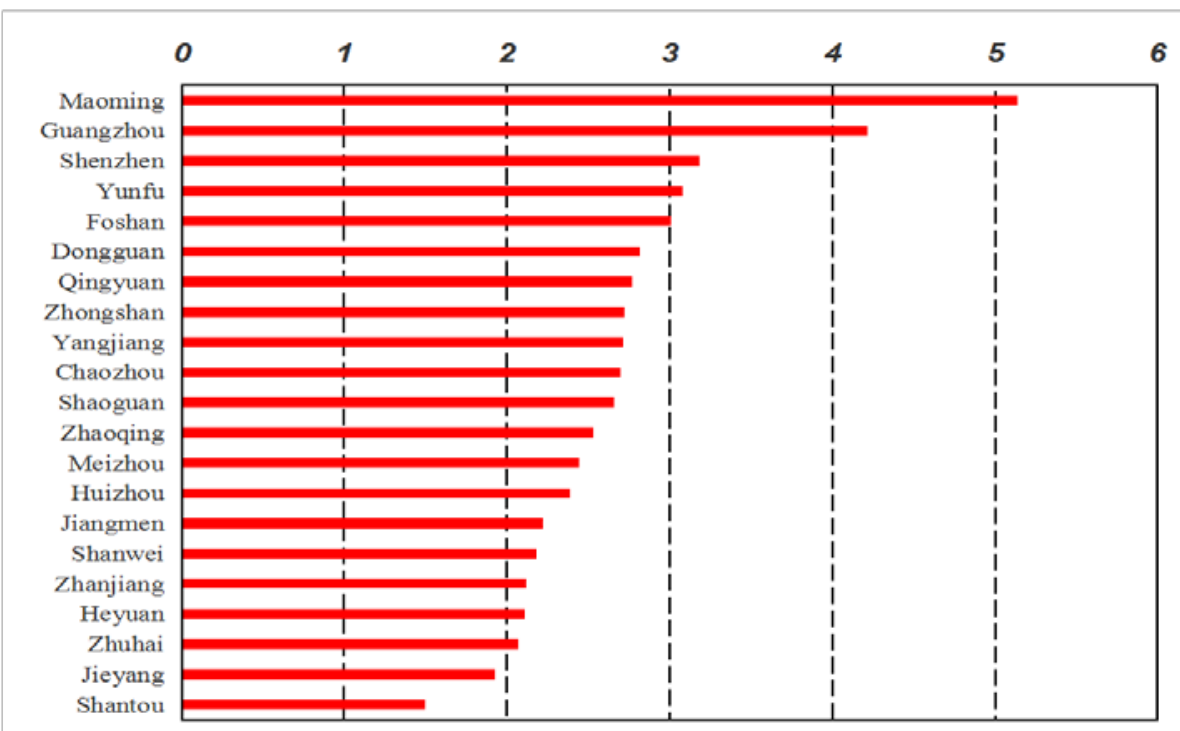

Fig. 1. The average value of the GDP recovery capability index. 
As shown in Figure 1, The GDP recovery index of Maoming, Guangzhou, Shenzhen, Yunfu and Foshan is more than 3, indicating that they are cities with strong GDP recovery capability. Maoming's leading position is attributed to its huge petrochemical industry and the driving role of relevant industrial chains. According to incomplete statistics in 2019, the output value of Maoming's petrochemical industry has reached more than 160 billion yuan, accounting for about half of Maoming's GDP. Guangzhou's high recovery capability is due to the strong foundation of modern manufacturing and service industries, such as GAC, Guangzhou Pharmaceutical, Hitachi Elevator and other leading enterprises, as well as the huge government, hospitals, universities, commerce and other modern service industries. Shenzhen's high recovery capability benefits from high-end manufacturing and modern service industries, such as Huawei, BYD, Vanke, Tencent and other leading enterprises in their industry. Yunfu and Foshan are adjacent to Guangzhou, which forms a good industrial base under the economic radiation of Guangzhou. (As the GDP recovery index of other cities is lower than 3, they are cities with low GDP recovery capability. Therefore, detailed analysis will not be made on them).

\subsubsection{The fiscal revenue recovery capability}

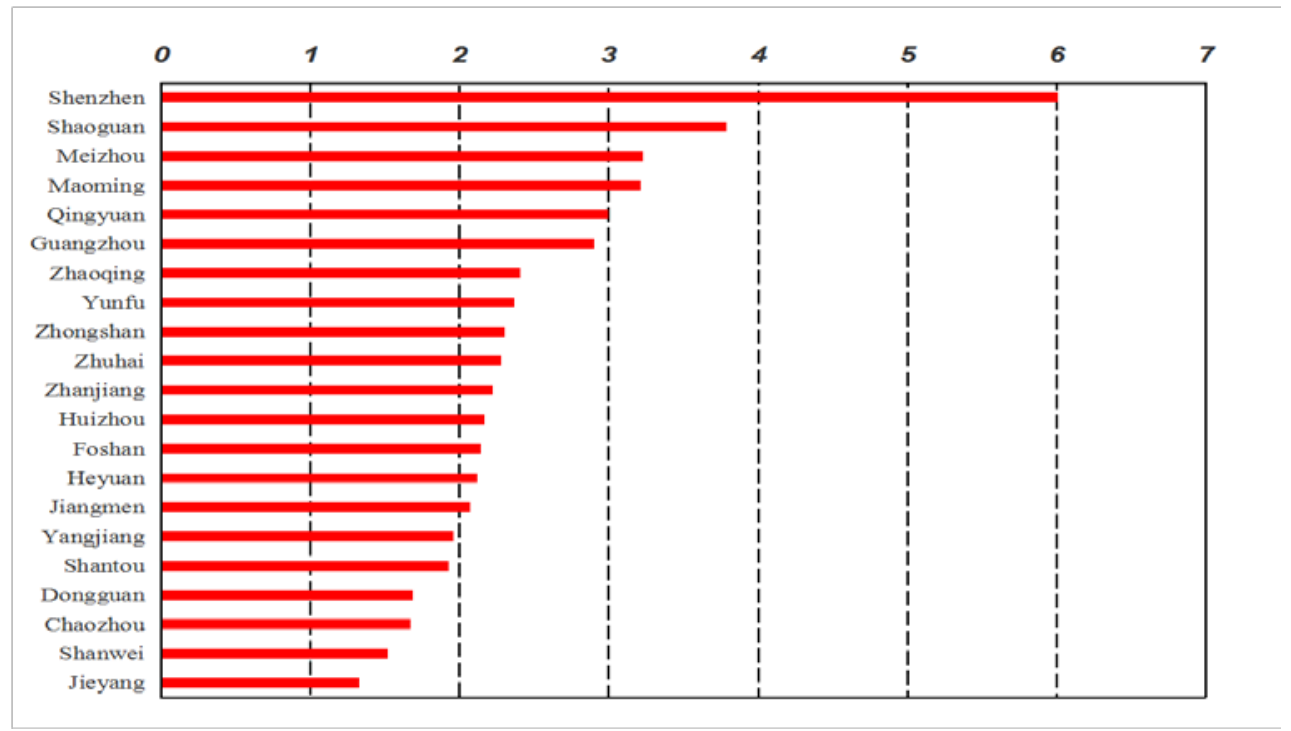

Fig. 2. The average value of the fiscal revenue recovery capability index.

As shown in Figure 2, The fiscal revenue recovery index of Shenzhen, Shaoguan, Meizhou, Maoming and Qingyuan is more than 3, indicating that they are cities with strong fiscal revenue recovery capability. It is worth mentioning that the fiscal revenue recovery index of Shenzhen is significantly ahead of other cities. This is because Shenzhen has continued to optimize and upgrade the industrial structure in recent years, which keeps the industrial competitiveness and profitcapability at a high level. The reason for the high fiscal revenue index of Shaoguan is related to the prosperity of the local upstream industries such as iron and steel metallurgy in recent years. The reason for the high fiscal revenue recovery index of Meizhou is related to the low labor cost of local enterprises, which promotes the enterprises to maintain a good profit situation. The recovery capability of Maoming remains at a high level because of the scale efficiency of its petrochemical industry. Qingyuan has become an important leisure tourism base due to the huge urban spillover effect of Guangzhou. (As the fiscal revenue recovery index of other cities is lower than 3, they are 
cities with low fiscal revenue recovery capability. Therefore, detailed analysis will not be made on them).

\section{Conclusion}

Taking the economic recovery capacity as the research object, this paper draws the following conclusions through econometric model and empirical research:

Firstly, the RCI model constructed in this paper has the characteristics of simple form, clear economic significance and convenient solution. It has good measurement function and can be used as a powerful tool to deal with all kinds of big data information.

Secondly, local governments should give priority to Shaoguan, Yangjiang, Zhuhai, Qingyuan, Shanwei and Shenzhen to take effective stimulus measures to achieve an efficient recovery process of GDP and fiscal revenue.

Thirdly, under the current situation that the epidemic continues in China, this study has practical significance for local governments to make precise policies and effectively promote the process of economic recovery, and has far-reaching significance for post-epidemic reconstruction in other countries and regions in the world.

Project of Guangdong Local Public Finance Research Center of Guangdong University of Finance \& Economics (20190007); Guangdong Education Science Planning Project (2018GXJK053).

\section{References}

1. [1] Banker R D,Charnes A and Cooper W W. Some Models for Estimating Technical and Scale Inefficiencies in Data Envelopment Analysis[J]. Management Science, 1984(9): 1078-1092.

2. Bei Jin. On Organizational Capital and Organizational Policy of the Economy: Enlightenment from the Epidemic Situation of COVID-19[J]. China Industrial Economics, 2020(4): 23-41.

3. Charnes A, Cooper W W and Rhodes E. Measuring the Efficiency of Decision Making Units[J]. European Journal of Operational Research, 1978(6): 429-444.

4. Charnes A, Cooper W W, Wei Q L et al. Cone Ratio Date Envelopment Analysis and Multi-Programming[J]. International Journal of Systems Science, 1987(7): 1099-1118.

5. Cullinane K, Song D W, Ji P, et al. An applicationg of DEA Windows Analysis to Container Port Pructiong Efficiency[J]. Review of Network Economics, 2004(2): 184206.

6. Gengzhong Feng, Yangyang Sun. The Influence of COVID-19 on the Economy and Society from the Perspective of Supply Chain[J]. Journal of Xi'an Jiaotong University (Social Sciences), 2020(4) : 42-49.

7. Haixia Lan, Xueyan Zhao. Spatial-Temporal Evolution and Innovation Environment Factors of Regional Innovation Efficiency in China[J]. Economic Geography, 2020(2): 97-107.

8. Lovell C A K. The Decomposition of Malmquist Productivity Indexes[J]. Journal of Productivity Analysis, 2003(20): 437-458.

9. Qiaobin Feng, Bo Han. The Effects of COVID-19 Epidemic on China's Finance and Economy and Relevant Countermeasures[J]. Public Finance Research, 2020(4): 3-22.

10. Seiford L M, Thrall R M. Recent Development in DEA, the Mathematical Programming Approach to Frontier Analysis[J]. Journal of Econimics, 1990(1/2): 7-38. 
11. Sengupta J K. Adynamic Efficiency Model Using Data Envelopment Analysis[J]. International Journal of Production Economics, 1999(3): 209-218.

12. Sengupta J K. Data Envelopment Analysis for Efficiency Measurement in the Stochastic Case[J]. Compiters \& Operations Research, 1987(2): 117-129.

13. Yangjie Wang, Xiangbo Tang, Xiaohong Chen. Development Opportunities and Strategies for the Digital Economy Industry in China under the Influence of new Coronavirus Pneumonia[J]. Science Research Management, 2020(6): 157-171.

14. Zhanxin Ma, Jiafeng Zhao. The efficiency antinomy of the DEA method and the short tail phenomenon of data[J]. Systems Engineering - Theory \& Practice, 2019(1): 200214.

15. Ziqiang Xin, Zhe Li, Zhixu Yang. People's Economic Confidence, Financial Values and Willingness to Expend during the Outbreak of COVID-19 in China[J]. Journal of Central University of Finance \& Economics, 2020(6): 118-128. 